\title{
Learning beyond the operating room: a call for feedback on postoperative care
}

\author{
Nayema Khan, MD $\cdot$ Ariel Mueller, MA $\cdot$ John D. Mitchell, MD • \\ Somnath Bose, MD
}

Received: 23 January 2018/Revised: 10 April 2018/Accepted: 11 April 2018/Published online: 9 May 2018

(C) Canadian Anesthesiologists' Society 2018

\section{To the Editor,}

Intraoperative anesthetic management has implications beyond the operating room. ${ }^{1}$ In most academic medical centres, a trainee's encounter with a patient is typically limited to the intraoperative period, concluding with signout to postanesthesia care unit (PACU) staff. When signout has been completed, trainees return to the operating room to care for the next patient on their schedule. This workflow focused on efficiency offers little opportunity for residents to obtain feedback on their intraoperative anesthetic management. We designed a survey to elucidate practices and perceptions of postoperative care and feedback among anesthesia trainees and PACU nursing staff with specific focus on identifying gaps in postoperative follow-up.

Between 20 September and 15 October 2016, all anesthesiology residents and PACU nursing staff at Beth Israel Deaconess Medical Center, Boston, MA, USA, were invited to participate in the online survey. This survey was performed as the first step of a larger project aimed at creating an effective electronic perioperative feedback tool. The Institutional Committee on Clinical Investigations

Electronic supplementary material The online version of this article (https://doi.org/10.1007/s12630-018-1147-6) contains supplementary material, which is available to authorized users.

N. Khan, MD · A. Mueller, MA · J. D. Mitchell, MD

S. Bose, MD $(\bowtie)$

Department of Anesthesia, Critical Care and Pain Medicine,

Beth Israel Deaconess Medical Center, Harvard Medical School,

Boston, MA, USA

e-mail: somnathbose@gmail.com

N. Khan, MD

Department of Anesthesiology, Kansas University Medical

Center, Kansas University, Kansas City, KS, USA determined the survey exempt from review. Participation was voluntary, with no incentives, and anonymity of responses was maintained. Topics addressed include PACU handoff, knowledge of a patient's PACU course, and patient follow-up practices (Supplement; available as Electronic Supplementary Material). Study data were collected and managed using the Research Electronic Data Capture survey tool. Responses are presented as descriptive statistics and median [interquartile range (IQR)]. SAS 9.3 (SAS Institute Inc., Cary, NC, USA) was used for all analyses.

Thirty-two residents (62\%) and 32 nurses (53\%) completed the survey. Perceptions of residents and nurses are presented in the Table. Residents more favorably perceived their awareness of postoperative pain control than the nurses with whom they worked. Both residents' and nurses' responses indicate that residents do not routinely follow up with patients in the PACU, either in person or electronically. Residents were unaware of the amount of pain medications or anti-emetics their patients required. They were unaware of whether their patient triggered a rapid response or experienced clinical delays in discharge from PACU. Residents however strongly agreed that this knowledge could influence their future anesthetics. Residents indicated that the workflow pattern served as a barrier to postoperative follow-up. Anesthesia trainees agreed strongly (90 [69-99] \%) that they were interested in receiving automated feedback on their patients' PACU course.

Participant responses indicate that workflow was perceived by residents as a barrier to follow-up. Residents disagreed with statements regarding their knowledge of their patients' postoperative care. Our findings suggest that trainees get a fragmented picture of their patients' perioperative course and call for focusing 
Table Resident and nursing perception of postoperative care

\begin{tabular}{|c|c|c|}
\hline & $\begin{array}{l}\text { Nurses } \\
n=32\end{array}$ & $\begin{array}{l}\text { Residents } \\
n=32\end{array}$ \\
\hline \multicolumn{3}{|l|}{ Agreement with the following } \\
\hline There is often a communication/information gap when a patient is handed off in the PACU & $58[39-72]$ & $50[25-62]$ \\
\hline A formal PACU handoff tool would be useful to assure adequate sign-out in the PACU & $52[50-75]$ & $60[40-78]$ \\
\hline Residents know how well they have controlled the patient's pain at the time of handoff in the PACU & $46[31-63]$ & $68[33-82]$ \\
\hline Residents know how well they have controlled the patient's nausea is at the time of handoff & $60[48-70]$ & $42[25-80]$ \\
\hline Residents know how long the typical PACU length of stay is & $36[22-50]$ & $21[4-39]$ \\
\hline Residents are aware of any clinical delays that affect a patient's transfer out of the PACU & $29[20-47]$ & $20[1-38]$ \\
\hline Residents routinely follow up with patients while they are still in the PACU & $21[15-36]$ & $34[19-62]$ \\
\hline \multicolumn{3}{|l|}{ Resident specific questions } \\
\hline \multicolumn{2}{|l|}{ I generally know how much pain medication my patient requires in the PACU } & $20[10-37]$ \\
\hline \multicolumn{2}{|l|}{ I generally know if my patient requires additional anti-emetics in the PACU } & $26[6-42]$ \\
\hline \multicolumn{2}{|l|}{ I know if my patient triggers in the PACU } & 25 [7-45] \\
\hline \multicolumn{2}{|l|}{ My work flow during a typical day allows me to follow up on my patient's in the PACU } & $23[2-35]$ \\
\hline \multicolumn{2}{|l|}{ I try to follow up with my patients in person when they are admitted as an inpatient } & $35[16-54]$ \\
\hline \multicolumn{2}{|l|}{ I use the PACU tabs in the perioperative and electronic medical record to follow up on my patient's PACU stay } & $31[0-66]$ \\
\hline \multicolumn{2}{|l|}{ Knowing the medications administered in the PACU would influence my anesthetic management } & 80 [70-93] \\
\hline \multicolumn{2}{|c|}{$\begin{array}{l}\text { Knowing the clinical delays that keep my patient from being transferred from the PACU would influence my anesthetic } \\
\text { management }\end{array}$} & $76[65-88]$ \\
\hline \multicolumn{2}{|l|}{ I would be interested in receiving feedback on clinical delays affect my patient's transfer out of the PACU } & $91[75-100]$ \\
\hline \multicolumn{2}{|l|}{ I would like to get regular automated feedback on the PACU course of my patients } & 90 [69-99] \\
\hline
\end{tabular}

Results are presented as median [interquartile range]

*Participants asked to agree with the statements indicated. Scores anchored at $0=$ strongly disagree, $50=$ neutral, and $100=$ strongly agree. PACU $=$ postanesthesia care unit

our attention on this aspect of perioperative care. We acknowledge that our study has multiple limitations including a single-centre design, a modest response rate, and likely response bias. The variance in responses of residents and nurses potentially indicates a wide variety of practice patterns or difficulty with the generalizability of the survey questions. Although the survey was centred around our institutional practices, we argue that the trainees' perceptions about lack of feedback surrounding PACU care may be representative of a larger gap in resident training. The few feedback tools designed for anesthesia trainees have focused exclusively on intraoperative management. ${ }^{2,3}$ Given the strong interest in receiving postoperative feedback, trainee engagement in building and implementing an automated tool could be considered a pragmatic next step in addressing this knowledge gap.

Acknowledgement Statistical support was provided by the Center for Anesthesia Research Excellence (CARE) at Beth Israel Deaconess Medical Center.

Conflicts of interest None declared.
Editorial responsibility This submission was handled by Dr. Gregory L. Bryson, Deputy Editor-in-Chief, Canadian Journal of Anesthesia.

Funding None.

\section{References}

1. Sessler DI. Long-term consequences of anesthetic management. Anesthesiology 2009; 111: 1-4.

2. Ehrenfeld JM, McEvoy MD, Furman WR, Snyder D, Sandberg WS. Automated near-real-time clinical performance feedback for anesthesiology residents: one piece of the milestones puzzle. Anesthesiology 2014; 120: 172-84.

3. Tanaka P, Bereknyei Merrell S, Walker K, et al. Implementation of a needs-based, online feedback tool for anesthesia residents with subsequent mapping of the feedback to the ACGME milestones. Anesth Analg 2017; 124: 627-35. 